\title{
Total Knee Arthroplasty after Anterior Cruciate Ligament Reconstruction
}

\author{
Matthew J. Best, MD ${ }^{1}$ Raj M. Amin, MD ${ }^{1} \quad$ Micheal Raad, MD ${ }^{1} \quad$ R. Timothy Kreulen, MD ${ }^{1}$ \\ Farah Musharbash, MD ${ }^{1}$ Daniel Valaik, MD ${ }^{1}$ John H. Wilckens, MD ${ }^{1}$ \\ ${ }^{1}$ Department of Orthopaedic Surgery, The Johns Hopkins University \\ School of Medicine, Baltimore, Maryland \\ J Knee Surg 2022;35:844-848. \\ Address for correspondence Matthew J. Best, MD, Department of \\ Orthopaedic Surgery, The Johns Hopkins University, 601 North \\ Caroline Street, 5th Floor, Baltimore, MD 21287 \\ (e-mail: mattbest8@gmail.com).
}

\author{
Abstract \\ Keywords \\ - anterior cruciate \\ ligament \\ reconstruction \\ - total knee \\ arthroplasty \\ - osteoarthritis
}

The incidence of anterior cruciate ligament (ACL) reconstruction is increasing in the United States, particularly in the older athlete. Patients who undergo ACL reconstruction are at higher risk for undergoing total knee arthroplasty (TKA) later in life. TKA in patients with prior $A C L$ reconstruction has been associated with longer operative time due in-part to difficulty with exposure and retained hardware. Outcomes after TKA in patients with prior $A C L$ reconstruction are not well defined, with some reports showing increased rate of complications and higher risk of reoperation compared with routine primary TKA, but these results are based on small and nonrandomized cohorts. Future research is needed to determine whether graft choice or fixation technique for $\mathrm{ACL}$ reconstruction influences outcomes after subsequent TKA. Furthermore, whether outcomes are affected by choice of TKA implant design for patients with prior ACL reconstruction warrants further study. This review analyzes the epidemiology, operative considerations, and outcomes of TKA following ACL reconstruction.
The anterior cruciate ligament $(\mathrm{ACL})$ provides rotational and translational stability to the knee joint. Patients with ACL deficient knees are at higher risk for developing meniscal and chondral injuries. ${ }^{1,2}$ The incidence of ACL tears and ACL reconstruction is increasing in the United States with over 100,000 ACL reconstructions performed each year. ${ }^{3,4}$ Some studies suggest that even in the presence of ACL reconstruction, patients may be at increased risk of developing osteoarthritis in the patellofemoral and tibiofemoral joints, especially in the setting of meniscal injury. ${ }^{5,6}$ Leroux et al matched 30,277 patients who underwent cruciate ligament reconstruction with individuals from the general population and showed that those with prior cruciate ligament reconstruction were seven times more likely to undergo total knee arthroplasty (TKA) than control patients 15 years postoperatively. ${ }^{7}$ Brophy et al evaluated 1,286 patients who underwent total knee arthroplasty and examined the effect of prior knee surgery on age at the time of TKA. ${ }^{8}$ The authors showed that patients who had previous knee surgery, especially prior

received

April 6, 2020

accepted after revision

October 5, 2020

published online

November 26, 2020

ACL reconstruction, underwent TKA at a significantly younger age by approximately 16 years. ${ }^{8}$

Studies assessing outcomes of TKA in patients with prior ACL reconstruction have shown mixed results. ${ }^{9}$ Watters et al studied 122 patients with prior ACL reconstruction who underwent TKA and showed a significantly longer operative time and five times greater risk of reoperation in the group with prior $\mathrm{ACL}$ reconstruction compared with the control group at 3-year follow-up. ${ }^{10}$ In contrast, Lizaur-Utrilla et al performed a matched case-control analysis of 37 patients with and without prior ACL reconstruction who underwent TKA and assessed outcomes at minimum 5-year follow-up. ${ }^{11}$ The authors concluded that although TKA in patients who had prior ACL reconstruction had increased technical difficulty and longer operative time, no differences in functional or radiologic outcomes scores were noted. ${ }^{11}$ Additionally, in a study of 35 patients with prior ACL reconstruction who underwent TKA, Hoxie et al showed no significant difference in postoperative range of motion, functional scores,

(c) 2020. Thieme. All rights reserved. Thieme Medical Publishers, Inc., 333 Seventh Avenue, 18th Floor, New York, NY 10001, USA
DOI https://doi.org/ 10.1055/s-0040-1721423. ISSN 1538-8506. 
periprosthetic infection, or rate of revision surgery compared with patients without prior ACL reconstruction. ${ }^{12}$

In this study, we review the epidemiology, treatment considerations, and outcomes of TKA following ACL reconstruction. Specifically, we examine the association between ACL reconstruction, osteoarthritis, and need for TKA. For patients requiring TKA after ACL reconstruction, we discuss technical aspects of surgery, including graft and implant choice. Functional outcomes, complications, and reoperation rate are also analyzed. Finally, we present avenues of future research in this patient population.

\section{Epidemiology}

\section{Anterior Cruciate Ligament Reconstruction and Osteoarthritis}

Over 100,000 ACL reconstructions are performed each year in the United States and the number of procedures continues to increase, especially in older patients where osteoarthritis and eventual TKA is more common., ${ }^{3,4}$ Mall et al studied trends in ACL reconstruction in the United States and showed that ACL reconstruction performed in patients 40 years or older increased from 1.65 per 100,000 person-years to 7.57 per 100,000 person-years from 1994 to $2006 .^{4}$

Osteoarthritis after ACL reconstruction is common, with up to $29 \%$ of patients developing moderate-to-severe osteoarthritis 20 years after ACL reconstruction..$^{13}$ Development of osteoarthritis after ACL reconstruction is multifactorial. In a study of 423 knees at minimum of 20-year follow-up after ACL reconstruction, Shelbourne et al identified older age at the time of ACL surgery, medial meniscectomy, and knee extension loss as predictors of development of osteoarthritis. ${ }^{13}$ Additional factors for the development of osteoarthritis after ACL reconstruction include cartilage injuries and loss of range of motion, which highlights the importance of physical therapy and motion exercises after ACL surgery. ${ }^{14,15}$ Finally, trauma to the articular cartilage during initial impact, chronic alterations in joint loading patterns, and possible genetic influences may also lead to osteoarthritis in these patients. ${ }^{16}$

\section{Total Knee Arthroplasty after Anterior Cruciate Ligament Reconstruction}

The incidence and prevalence of TKA has also steadily risen over the past 20 years. Over 600,000 TKAs are performed per year in the United States and this number is predicted to increase by over $140 \%$ by $2050 .{ }^{17}$ Few studies exist investigating the incidence and epidemiology of patients undergoing TKA after prior ACL reconstruction. In a population-based matched cohort study, Leroux et al compared patients who underwent cruciate ligament reconstruction ( $>98 \%$ of which were ACL reconstruction) from 1993 to 2008 and matched them with individuals from the general population in Canada who did not have prior knee surgery. ${ }^{7}$ They found a significant difference in event rate between the matched case and control cohorts with the cruciate reconstruction cohort undergoing TKA at a rate of 0.68 per 1,000 person-years compared with 0.10 per 1,000 person-years in the control cohort. ${ }^{7}$ They also showed that 15 years after cruciate ligament reconstruction, there was significant difference in the cumulative incidence of TKA between the cruciate reconstruction cohort at $1.4 \%$ compared with the control cohort at $0.2 \%{ }^{7}$ Demographic factors of patients undergoing TKA after $A C L$ reconstruction have been investigated in several studies. In their study of 122 patients with prior ACL reconstruction at the time of TKA, Watters et al showed a mean age 58 years, with $55 \%$ being male. ${ }^{10}$ In a smaller study of 37 patients undergoing TKA, Lizaur-Utrilla et al found a mean age of 69.6 years with $60 \%$ being male. ${ }^{11}$

\section{Operative Considerations}

\section{Exposure/Intraoperative Difficulty}

Surgical exposure for TKA may be more difficult in patients with prior surgery. Lizaur-Utrilla et al compared intraoperative findings at the time of TKA between 37 patients with prior ACL reconstruction and compared them to patients without prior ACL reconstruction. ${ }^{11}$ The authors found that in the prior ACL reconstruction group, there were more cases with tibial rotational and translational deformity that caused difficulty with ligamentous balance. ${ }^{11}$ Overall, they found that technical difficulties were encountered in 26 of 37 knees in the ACL reconstruction group including partial patellar tendon avulsion $(n=1)$, need for quadriceps snip $(n=1)$, additional medial and posterior capsular release $(n=24)$, need for increased thickness of polyethylene insert $(n=15)$, and need for tibial stem $(n=5)$. In the control group, only two cases of required increased thickness of polyethylene insert and no other technical difficulties were encountered. In this study, the authors noted that the main difficulty in most patients with prior ACL reconstruction was due to varus deformity and retraction of the medial soft tissues which required added medial release. ${ }^{11}$ In a study of 22 patients with prior ACL reconstruction, Magnussen et al also reported difficulty with tibial exposure. They showed that three patients required tibial tubercle osteotomy in the ACL reconstruction group compared with 0 patients in the matched-control group. ${ }^{18}$

In contrast, Hoxie et al showed no added technical difficulties in a cohort of 35 patients (36 TKAs) with prior ACL reconstruction. ${ }^{12}$ Additionally, they showed that no stems or augments were required during the TKA procedure. Although patella baja has been associated with prior ACL reconstruction with patella tendon autograft, the authors noted that no patients with prior ACL reconstruction with patella tendon autograft had evidence of patella baja at the time of TKA. ${ }^{19-21}$ Three patients with prior ACL reconstruction with hamstring autograft and one patient with unknown graft type did have patella baja at the time of TKA. ${ }^{12}$

\section{Retained Hardware}

Removal of prior implants or hardware at the time of TKA may be a concern in patients with prior knee surgery. In a retrospective cohort of 22 patients undergoing TKA after prior ACL reconstruction, Magnussen et al reported the need for removal of metallic hardware related to the prior ACL 
reconstruction in $45 \%$ of patients. ${ }^{18}$ Watters et al studied 122 patients undergoing TKA after prior ACL reconstruction and found that $50 \%$ of patients required implant removal at the time of TKA. ${ }^{10}$ The authors highlight the difficulty with retained hardware at the time of TKA as the implants may be covered with cortical bone and prevent placement of the TKA components. Specifically, interference screws or staples may be challenging during the intercondylar notch cut for posterior stabilized components or during drilling for the tibial stem. ${ }^{10}$ The authors recommend removal with a screwdriver if accessible or carbide burr if needed. Newer hardware for ACL reconstruction such as biocomposite interference screws and suspensory fixation with continuous loop endobutton may not lead to the same difficulty or increased operative time as other fixation methods for ACL reconstruction, but larger cohorts are needed to verify this.

\section{Operative Time}

Operative time for TKA may be increased in patients with prior ACL reconstruction due to difficulty with exposure, technical difficulty with ligament balancing, or due to removal of prior implants, but this has not been associated with increased blood loss. $^{10,22}$ In a retrospective study, Chong et al analyzed 101 patients undergoing TKA after prior ACL reconstruction. ${ }^{22} \mathrm{Al}$ though there was no difference in blood loss between patients with and without prior ACL reconstruction, the authors found a statistically significant difference in mean operative time in patients who required tibia hardware removal ( $74 \pm 23$ minutes vs. control: $64 \pm 21$ minutes) and patients who required tibia and femur hardware removal $(79 \pm 24$ minutes vs. control: $65 \pm 19$ minutes). ${ }^{22}$ The authors findings allude to hardware removal being the main contributor to operative time, especially hardware removed from the tibia. Similar findings were demonstrated in the Watters et al study, where $50 \%$ of 122 patients required hardware removal and mean operative time was significantly longer in this group ( 88 minutes) compared with controls (73 minutes).$^{10}$ Lizaur-Utrilla et al also found a longer mean operative time for patients with prior ACL reconstruction but attributed this to increased technical difficulty during surgery requiring additional soft tissue release and ligament balancing, unlike the aforementioned studies where hardware removal was the listed contributor. ${ }^{11}$ In contrast, Magnussen et al did not find a significant difference in mean operative time between patients with and without prior ACL reconstruction despite the need for removal of retained hardware in $45 \%$ of patients. ${ }^{18}$ It is possible, however, that with only 22 patients in each group, this study may have been underpowered to find a statistically significant difference $(84 \pm 21$ minutes in the study group compared with $75 \pm 17$ minutes in controls, $p=0.13$ ). ${ }^{18}$

\section{Outcomes}

\section{Functional Outcomes}

Numerous groups have analyzed postoperative functional scores in patients undergoing TKA after ACL reconstruction and have shown similar results compared with patients who have not had prior ACL reconstruction. In a retrospective study of 37 patients who underwent TKA after prior ACL reconstruction, Lizaur-Utrilla at al found no significant differences in functional scores, patient satisfaction scores, or postoperative range of motion compared with controls. ${ }^{11}$ Similarly, Watters et al in their cohort of 122 patients with prior ACL reconstruction found no significant difference in range of motion, pain, or functional scores after TKA compared with controls. ${ }^{10}$ In contrast, Magnussen et al showed that the rate of postoperative stiffness was significantly higher in patients with prior ACL reconstruction, although there were no differences in final range of motion or outcome scores 2 to 3 years postoperatively. ${ }^{18}$ Overall, prior ACL reconstruction seems to have no significant effect on postoperative functional outcomes after TKA, but larger studies are needed to find more subtle differences.

\section{Complications/Infection}

Complication rates in patients undergoing TKA after ACL reconstruction have been reported at higher rates than in routine primary TKA. ${ }^{10}$ In their review, Watters et al showed the development of prosthetic infection in four patients who had $\mathrm{ACL}$ reconstruction prior to TKA (3.3\%) compared with no infections in the control group. ${ }^{10}$ Two of the four prosthetic infections occurred within 1 year of TKA and the other two infections occurred at 22 to 33 months. All infections resolved with irrigation, debridement, liner exchange, and antibiotics. Other reports present conflicting data on the effect of prior surgery on infection rate after TKA with no significant difference in prosthetic infection rates between patients with and without prior ACL reconstruction. ${ }^{11,22}$ Additionally, conflicting reports exist for whether any prior knee surgery (such as osteotomy or fracture fixation) influences infection after TKA. ${ }^{23-25}$ It is important to consider confounders when analyzing infection after TKA. For instance, in the study by Chong et al, although no significant differences in prosthetic infection were seen between groups, the control group had a higher rate of diabetes (13\%) compared with the ACL group (8\%). No analysis was performed on baseline characteristics of either group and it is possible that this confounder of a higher diabetes rate in the control group may have offset any increased risk of infection from prior ACL reconstruction in the ACL group. Furthermore, longer operative time has been associated with increased risk of prosthetic joint infection after TKA. ${ }^{26,27}$ It is important to determine whether increased operative time alone portends a higher risk of infection in TKA patients with prior ACL reconstruction or if other factors such as need for excessive soft tissue release, bony loss or deformity, or presence of prior scarring might influence infection rates in patients with prior ACL reconstruction. Nonetheless, patients with ACL reconstruction who undergo TKA may be at higher risk for postoperative prosthetic infection. Larger, controlled studies are needed to validate this finding among broader patient populations.

\section{Reoperation}

Reoperation after TKA can impose significant costs to the health care system as well as contribute to patient burden and morbidity. In the study by Watters et al, reoperation was 
five times higher in the ACL group than in the control group. Aside from the four reoperations for prosthetic infection, there were seven other reoperations in the ACL group compared with only two reoperations in the control group. ${ }^{10}$ Three patients in the ACL group required arthroscopic synovectomy for painful patellar crepitus, three patients underwent manipulation under anesthesia for early postoperative arthrofibrosis, and one patient required revision for patellar button failure, polyethylene wear, and instability. In the control group, one patient required closure of a superficial wound dehiscence and one required manipulation under anesthesia for arthrofibrosis. Studies by Hoxie et al and Chong et al showed no significant differences in rates of reoperation after TKA between patients with and without prior history of ACL reconstruction. ${ }^{12,22}$ Additionally, Hoxie et al showed no significant difference in reoperation rate at 5 and 10 years postoperatively. ${ }^{12}$

\section{Future Research}

Several topics warrant further study for patients undergoing TKA after ACL reconstruction. The effect of ACL graft choice at the time of reconstruction on outcomes after TKA is not known. Devascularization and altered tendon remodeling may lead to extensor mechanism dysfunction after patellar tendon harvest for ACL reconstruction, and can occur up to 6 years after ACL reconstruction. ${ }^{28,29}$ In the Watters et al study, three patients in the ACL group required arthroscopic synovectomy for painful patellar crepitus after TKA. ${ }^{10}$ Due to the duration of time between ACL reconstruction and TKA, the authors did not have sufficient data to determine ACL graft type or technique and could not assess these variables.

It is unclear whether differences exist between cruciate retaining and posterior stabilized TKA designs in patients with prior ACL reconstruction. Altered knee biomechanics may exist in patients even after ACL reconstruction and further study is needed to determine if this influences outcomes after TKA. James et al analyzed 188 patients with prior ACL reconstruction and 35 patients with prior multiligament reconstruction who underwent TKA, and found they had a significantly higher use of constrained implants at the time of TKA when compared with controls without prior ligament reconstruction. ${ }^{30}$ The authors did not find any difference between groups in postoperative knee ROM, patientreported outcomes, or incidence of revision surgery. ${ }^{30}$

Finally, further study is needed to determine if differences between old and new ACL fixation implants have effect on subsequent TKA. Several studies show increased operative time for removal and increased technical difficulty due to retained hardware from prior ACL reconstruction. ${ }^{10,22}$ Biocomposite screws and suspensory fixation may be less challenging during TKA than metal interference screws but more studies are needed to assess this.

\section{Conclusion}

TKA in patients with prior ACL reconstruction has been associated with longer operative time, increased rate of prosthetic infection, and higher risk of reoperation compared with routine primary TKA. However, these results are based on small and nonrandomized cohorts. In contrast, several studies have demonstrated equivalent functional outcomes and no difference in technical difficulty in patients with prior ACL reconstruction undergoing TKA compared with patients without prior knee surgery. Further research is needed to determine the risk that prior ACL reconstruction has on future incidence of TKA as well as operative considerations and outcomes.

\section{Funding}

None.

Conflict of Interest

None declared.

\section{References}

1 Brambilla L, Pulici L, Carimati G, et al. Prevalence of associated lesions in anterior cruciate ligament reconstruction: correlation with surgical timing and with patient age, sex, and body mass index. Am J Sports Med 2015;43(12):2966-2973

2 Chhadia AM, Inacio MC, Maletis GB, Csintalan RP, Davis BR, Funahashi TT. Are meniscus and cartilage injuries related to time to anterior cruciate ligament reconstruction? Am J Sports Med 2011;39(09):1894-1899

3 Buller LT, Best MJ, Baraga MG, Kaplan LD. Trends in anterior cruciate ligament reconstruction in the United States. Orthop J Sports Med 2014;3(01):2325967114563664

4 Mall NA, Chalmers PN, Moric M, et al. Incidence and trends of anterior cruciate ligament reconstruction in the United States. Am J Sports Med 2014;42(10):2363-2370

5 Culvenor AG, Cook JL, Collins NJ, Crossley KM. Is patellofemoral joint osteoarthritis an under-recognised outcome of anterior cruciate ligament reconstruction? A narrative literature review. Br J Sports Med 2013;47(02):66-70

6 Neuman P, Englund M, Kostogiannis I, Fridén T, Roos H, Dahlberg LE. Prevalence of tibiofemoral osteoarthritis 15 years after nonoperative treatment of anterior cruciate ligament injury: a prospective cohort study. Am J Sports Med 2008;36(09):1717-1725

7 Leroux T, Ogilvie-Harris D, Dwyer T, et al. The risk of knee arthroplasty following cruciate ligament reconstruction: a population-based matched cohort study. J Bone Joint Surg Am 2014;96 (01):2-10

8 Brophy RH, Gray BL, Nunley RM, Barrack RL, Clohisy JC. Total knee arthroplasty after previous knee surgery: expected interval and the effect on patient age. J Bone Joint Surg Am 2014;96(10):801-805

9 Chaudhry ZS, Salem HS, Purtill JJ, Hammoud S. Does prior anterior cruciate ligament reconstruction affect outcomes of subsequent total knee arthroplasty? A systematic review. Orthop J Sports Med 2019;7(07):2325967119857551

10 Watters TS, Zhen Y, Martin JR, Levy DL, Jennings JM, Dennis DA. Total knee arthroplasty after anterior cruciate ligament reconstruction: not just a routine primary arthroplasty. J Bone Joint Surg Am 2017;99(03):185-189

11 Lizaur-Utrilla A, Martinez-Mendez D, Gonzalez-Parreño S, MarcoGomez L, Miralles Muñoz FA, Lopez-Prats FA. Total knee arthroplasty in patients with prior anterior cruciate ligament reconstruction. J Arthroplasty 2018;33(07):2141-2145

12 Hoxie SC, Dobbs RE, Dahm DL, Trousdale RT. Total knee arthroplasty after anterior cruciate ligament reconstruction. J Arthroplasty 2008;23(07):1005-1008

13 Shelbourne KD, Benner RW, Gray T. Results of anterior cruciate ligament reconstruction with patellar tendon autografts: objective factors associated with the development of osteoarthritis at 
20 to 33 years after surgery. Am J Sports Med 2017;45(12): $2730-2738$

14 Shelbourne KD, Freeman H, Gray T. Osteoarthritis after anterior cruciate ligament reconstruction: the importance of regaining and maintaining full range of motion. Sports Health 2012;4(01): 79-85

15 Shelbourne KD, Urch SE, Gray T, Freeman H. Loss of normal knee motion after anterior cruciate ligament reconstruction is associated with radiographic arthritic changes after surgery. Am J Sports Med 2012;40(01):108-113

16 Paschos NK. Anterior cruciate ligament reconstruction and knee osteoarthritis. World J Orthop 2017;8(03):212-217

17 Inacio MCS, Paxton EW, Graves SE, Namba RS, Nemes S. Projected increase in total knee arthroplasty in the United States: an alternative projection model. Osteoarthritis Cartilage 2017;25 (11):1797-1803

18 Magnussen RA, Demey G, Lustig S, Servien E, Neyret P. Total knee arthroplasty for secondary osteoarthritis following ACL reconstruction: a matched-pair comparative study of intra-operative and early post-operative complications. Knee 2012;19(04): 275-278

19 Salmon LJ, Russell VJ, Refshauge K, et al. Long-term outcome of endoscopic anterior cruciate ligament reconstruction with patellar tendon autograft: minimum 13-year review. Am J Sports Med 2006;34(05):721-732

20 Järvelä T, Paakkala T, Kannus P, Järvinen M. The incidence of patellofemoral osteoarthritis and associated findings 7 years after anterior cruciate ligament reconstruction with a bone-patellar tendon-bone autograft. Am J Sports Med 2001;29(01):18-24

21 Muellner T, Kaltenbrunner W, Nikolic A, Mittlboeck M, Schabus R, Vécsei V. Shortening of the patellar tendon after anterior cruciate ligament reconstruction. Arthroscopy 1998;14(06):592-596
22 Chong ACM, Fisher BT, MacFadden LN, Piatt BE. Prior anterior cruciate ligament reconstruction effects on future total knee arthroplasty. J Arthroplasty 2018;33(09):2821-2826

23 Farfalli LA, Farfalli GL, Aponte-Tinao LA. Complications in total knee arthroplasty after high tibial osteotomy. Orthopedics 2012; 35(04):e464-e468

24 Suzuki G, Saito S, Ishii T, Motojima S, Tokuhashi Y, Ryu J. Previous fracture surgery is a major risk factor of infection after total knee arthroplasty. Knee Surg Sports Traumatol Arthrosc 2011;19(12): 2040-2044

25 Klatte TO, Schneider MM, Citak M, et al. Infection rates in patients undergoing primary knee arthroplasty with pre-existing orthopaedic fixation-devices. Knee 2013;20(03):177-180

26 Duchman KR, Pugely AJ, Martin CT, Gao Y, Bedard NA, Callaghan JJ. Operative time affects short-term complications in total joint arthroplasty. J Arthroplasty 2017;32(04):1285-1291

27 Peersman G, Laskin R, Davis J, Peterson MG, Richart T. Prolonged operative time correlates with increased infection rate after total knee arthroplasty. HSS J 2006;2(01):70-72

28 Marumoto JM, Mitsunaga MM, Richardson AB, Medoff RJ, Mayfield GW. Late patellar tendon ruptures after removal of the central third for anterior cruciate ligament reconstruction. A report of two cases. Am J Sports Med 1996;24(05): 698-701

29 Rosenberg TD, Franklin JL, Baldwin GN, Nelson KA. Extensor mechanism function after patellar tendon graft harvest for anterior cruciate ligament reconstruction. Am J Sports Med 1992;20 (05):519-525, discussion 525-526

30 James EW, Blevins JL, Gausden EB, et al. Increased utilization of constraint in total knee arthroplasty following anterior cruciate ligament and multiligament knee reconstruction. Bone Joint J 2019;101-B(7_Supple_C):77-83 